\title{
Modeling and Decoupling Control of the CoaX Micro Helicopter
}

\section{Conference Paper}

Author(s):

Fankhauser, Péter; Bouabdallah, Samir; Leutenegger, Stefan; Siegwart, Roland

Publication date:

2011

Permanent link:

https://doi.org/10.3929/ethz-a-010025759

Rights / license:

In Copyright - Non-Commercial Use Permitted

Originally published in:

https://doi.org/10.1109/IROS.2011.6094745 


\title{
Modeling and Decoupling Control of the CoaX Micro Helicopter
}

\author{
Péter Fankhauser, Samir Bouabdallah, Stefan Leutenegger, Roland Siegwart
}

\begin{abstract}
The dynamics of micro coaxial helicopters are coupled, especially in the presence of a stabilizer bar and in dynamic maneuvers. This paper presents a model-based approach for active decoupling of the dynamics of a micro coaxial helicopter. This allows for easier and more accurate operation of the system. The nonlinear model covers all degrees of freedom for attitude and altitude. It accounts for hover and cruise flight situations and explicitly captures the off-axis dynamics and the dynamics of the stabilizer bar. A six-axis force/torque sensor and an RPM measurement system are used in a custom built test bench. It is applied for analysis of the forces and torques generated by the rotors in combination with the dynamics of the drive train and the swashplate. The parameter identification and the model validation is obtained with flight data recorded with a vision-based motion tracking system. The decoupling controller is implemented on the commercial robotic helicopter CoaX and its performance is shown via a motion experiment.

Index Terms - Coaxial micro helicopter, Decoupling control, Nonlinear model, Parameter identification
\end{abstract}

\section{INTRODUCTION}

Coaxial micro helicopters are suitable for autonomous indoor navigation. Their advantage is the small footprint thanks to their tailless design. In addition, they exhibit increased thrust force and efficiency given the same area compared to single- or quadrotor configurations.

Naturally, the dynamics of a helicopter are subject to cross coupling. Off-axis dynamics are stimulated due to effects such as mechanical misalignment of the cyclic input, and the different airflow on the rotor blades at high velocities. This leads to an increased complexity in the control of the helicopter. The work presented is this paper was conducted to approach this issue with a decoupling controller.

Different works have been performed in modeling of a micro helicopter. Schafroth [1] has presented a nonlinear model of a micro coaxial helicopter for all degrees of freedom. The model and parameter identification describe the system near hover and thus neglect important coupling sources. Mettler [2] has presented a linear model based on identification for both hover and cruise flight situations. The model describes a single rotor helicopter which is substantially larger then the CoaX. We base our model on [1] and extend it with approaches from [2] and our own development.

First, the CoaX helicopter is introduced in sect. II. The model of the CoaX is derived with all its relevant dynamics in sect. III. The parameter identification (sect. IV) is split into the identification on the test bench and the model validation.

\footnotetext{
The authors are with the Autonomous Systems Lab (ASL), ETH Zurich, Tannenstrasse 3, 8092 Zurich, Switzerland peterfalstudent.ethz.ch

The associated MATLAB/Simulink model is available online at http: //www.skybotix.com/.
}

Section V describes the model-based decoupling controller and shows results.

\section{The COAX Helicopter}

The CoaX is a miniature robotic helicopter with coaxial rotor setup designed for research and education (fig. 1). The system weighs about $300 \mathrm{~g}$ (depending on the configuration) and has a span of $340 \mathrm{~mm}$ and a height of $274 \mathrm{~mm}$. The rotors are driven by two brushless motors via a set of gears. The steering is provided by two servo motors controlling the swashplate of the lower rotor. The upper rotor is attached to the stabilizer bar acting as a passive regulator of the roll and pitch of the helicopter.

CoaX is equipped i.a. with an integrated Inertial Measurement Unit (IMU), a pressure sensor, a down-facing sonar, two microcontrollers and a Gumstix. For communication the Bluetooth or Zigbee module and a $2.4 \mathrm{GHz}$ remote control are used.

\section{DYNAMIC MODEL}

The requirement for the dynamic model is that all relevant dynamics at hover and especially at cruise conditions are reflected accurately. However, the model should be simple enough to be used for control and real-time simulations.

The model of the CoaX is based on the rigid body motion where all existing forces and torques result from different subsystems. These subsystems represent physically meaningful dynamics e.g. the stabilizer bar, the swashplate and the upper and lower drive train.

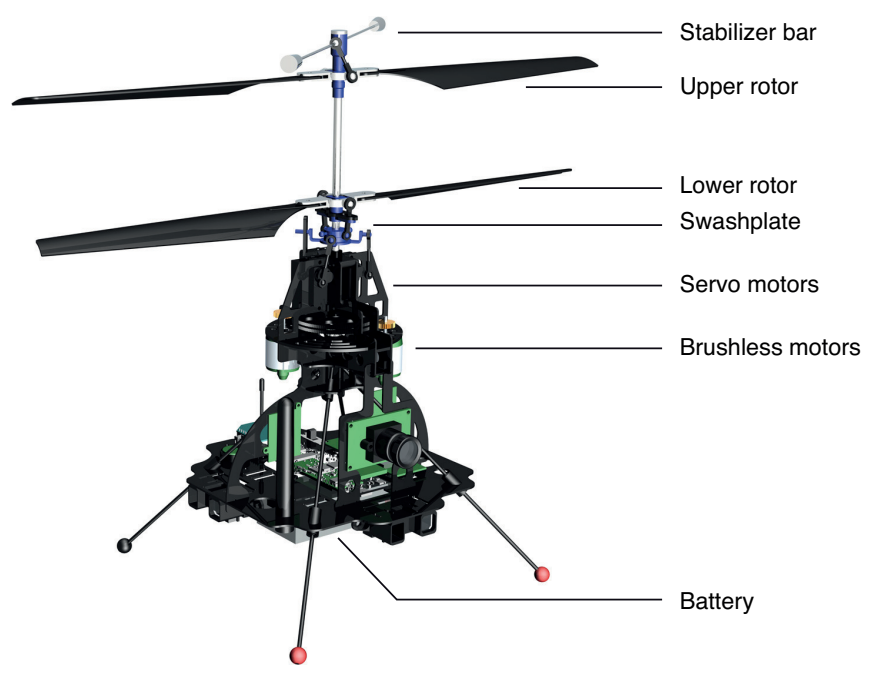

Fig. 1. CAD model of the CoaX 


\section{A. Coordinate Frames and Rigid-Body Dynamics}

We introduce an inertial reference frame $I$ and the body fixed frame $B$ placed in the Center of Gravity (CoG) of the helicopter. The dynamics of the body are described by the Newton-Euler equation for a single rigid body in the bodyfixed reference frame $B$. The equations of motion are derived and explained in [1]-[5].

\section{B. Forces and Torques}

The force vector $\mathbf{F}$ and the torque vector $\mathbf{M}$ acting on the body sum up as:

$$
\begin{aligned}
\mathbf{F}= & \mathbf{T}_{\text {up }}+\mathbf{T}_{\mathrm{lw}}+\mathbf{D}+\mathbf{G} \\
\mathbf{M}= & \mathbf{Q}_{\text {up }}+\mathbf{Q}_{\mathrm{lw}}+\mathbf{r}_{C, \text { up }} \times \mathbf{T}_{\text {up }}+\mathbf{r}_{C, \mathrm{lw}} \times \mathbf{T}_{\mathrm{lw}} \\
& +\mathbf{Q}_{\text {react,up }}+\mathbf{Q}_{\text {react,lw }}+\mathbf{M}_{\text {flap }, \text { up }}+\mathbf{M}_{\text {flap }, \mathrm{lw}} .
\end{aligned}
$$

The aerodynamic drag force on the fuselage due to the downwash of the rotors does not appear because the measurements of the thrust are made in combination with fuselage and are thus already included.

1) $\mathbf{T}$ - Rotor Thrust: We define the thrust of a rotor as $\mathbf{T}_{i}=T_{i} \cdot \mathbf{n}_{T, i}$ with $i \in\{1 \mathrm{w}, \mathrm{up}\}$ for the lower and upper rotor. The thrust magnitude $T_{i}$ can be defined as in [5]:

$$
T_{i}=c_{T, i} k_{T} \Omega_{i}^{2} \quad \text { with } \quad k_{T}=\pi \rho R^{4},
$$

and $\Omega_{i}$ being the rotational speed of the rotor. The factor $c_{T, i}$ denotes the thrust coefficient which is assumed to be constant (identified in coaxial setup at hover). We neglect the dynamic mutual interference of the rotors since only small changes $(\sim 1-3 \%)$ in the rotational velocity are necessary for yaw and height movement of the helicopter. Further, the influence of the horizontal and vertical velocity is neglected because usual body velocities are only a minor fraction of the speed of the downwash $(\sim 1-4 \%$ for vertical and $\sim 5-20 \%$ for horizontal velocities). The constant $k_{T}$ depends on the air density $\rho$ and the rotor radius $R$. The normalized direction of the thrust vector $\mathbf{n}_{T, i}$ is perpendicular to the Tip Path Plane (TPP) and can be expressed as a rotation $\alpha$ about the $\mathrm{x}$-axis and a rotation $\beta$ about the $y$-axis of the body frame [4].

2) $\mathrm{Q}$ - Rotor Drag Torque: Similarly to the rotor thrust, the rotor drag torque can be defined as $\mathbf{Q}_{i}=Q_{i} \cdot \mathbf{n}_{Q, i}$ with the drag torque magnitude

$$
Q_{i}=c_{Q, i} k_{Q} \Omega_{i}^{2} \quad \text { with } \quad k_{Q}=\pi \rho R^{5} .
$$

The rotor drag torque acts on the z-axis of the body and with opposed sign [1].

3) $\mathbf{Q}_{\text {react }}$ - Rotor Reaction Torque: The change of the rotational speeds of the rotors leads to a reaction torque on the fuselage $\mathbf{Q}_{\text {react }, i}=Q_{\text {react }, i} \cdot \mathbf{n}_{Q, \text { react }, i}$ with

$$
Q_{\text {react }, i}=J_{\text {rot }, i} \dot{\Omega}_{i}
$$

and $J_{\text {rot, } i}$ denoting the inertia of the rotating parts such as the rotor blades, the stabilizer bar, the motors and the gears. The direction is the same as for the rotor drag torque [1].
4) $\mathbf{r}_{C} \times \mathbf{T}-$ Thrust Induced Torques: The tilting of the TPP changes the direction of the thrust vector $\mathbf{T}_{i}$ which induces a torque calculated with $\mathbf{r}_{C, i} \times \mathbf{T}_{i}$, where $\mathbf{r}_{C, i}$ is the vector from the $\mathrm{CoG}$ to the hinges of the lower and upper rotor.

5) $\mathbf{M}_{\text {flap }}$ - Rotor Flapping Torque: The flapping of the rotor blades is caused by steering inputs (from the swashplate or stabilizer bar) and from horizontal velocities [6]. The two rotors have a lead-lag hinge but are hingless in the flapping direction. However, the elasticity of the blades allows for flapping motion. This leads to important torques on the roots of the blades which have to be considered in the model. As described in [5], the flapping torques can be simplified as a flapping hinge with a torsion spring which exerts a torque depending on the deflection of the TPP,

$$
\mathbf{M}_{\text {flap }, i}=k_{\mathrm{sprg}, i}\left[\begin{array}{c}
\alpha_{i} \\
\beta_{i} \\
0
\end{array}\right]
$$

with $k_{\mathrm{sprg}, i}$ denoting the blade stiffness.

6) D - Fuselage Drag: Velocities of the helicopter lead to drag on the fuselage which can be approximated by a velocity dependent torque and a force in the CoG. The drag force is written as

$$
\mathbf{D}=\left[\begin{array}{c}
-a_{\mathrm{Fx}, u} u^{2} \operatorname{sign}(u) \\
-a_{\mathrm{Fy}, v} v^{2} \operatorname{sign}(v) \\
0
\end{array}\right]
$$

Thanks to symmetry of the CoaX the influence of off-axis velocities is dropped. The drag force on the fuselage in the vertical direction is neglected because high velocities are mainly expected in the horizontal motion. The velocity dependent torque is modeled as an effect on the lower rotor and is described sect. III-C.

7) $\mathbf{G}$ - Gravity: Finally, the weight force $\mathbf{G}$ in the body reference system $B$ amounts to ${ }_{B} \mathbf{G}=\mathrm{A}_{B J} \cdot{ }_{J} \mathbf{G}$, [1].

\section{Dynamic Elements}

1) Upper Rotor and Stabilizer Bar: The stabilizer bar is lagging behind the roll/pitch motion of the fuselage due to its high inertia. It is linked to the upper rotor and therefore counteracts on sudden changes in roll/pitch through cyclic input on the upper rotor.

We define the deflection angles of the stabilizer bar relative to the inertial reference frame as $\eta_{\text {bar }}$ and $\zeta_{\text {bar }}$. The following characteristic on the roll/pitch motion can be modeled as first order element as in [1]:

$$
\dot{\eta}_{\text {bar }}=\frac{1}{\tau_{f, \text { up }}}\left(\phi-\eta_{\text {bar }}\right) \quad \text { and } \quad \dot{\zeta}_{\text {bar }}=\frac{1}{\tau_{f, \text { up }}}\left(\theta-\zeta_{\text {bar }}\right),
$$

with roll and pitch angles $\phi$ and $\theta$. The time constant $\tau_{f \text {,up }}$ covers the combined system of the stabilizer bar and the upper rotor. The angles of the stabilizer bar relative to the body reference frame are obtained by

$$
\alpha_{\mathrm{bar}}=\eta_{\mathrm{bar}}-\phi \quad \text { and } \quad \beta_{\mathrm{bar}}=\zeta_{\mathrm{bar}}-\theta
$$




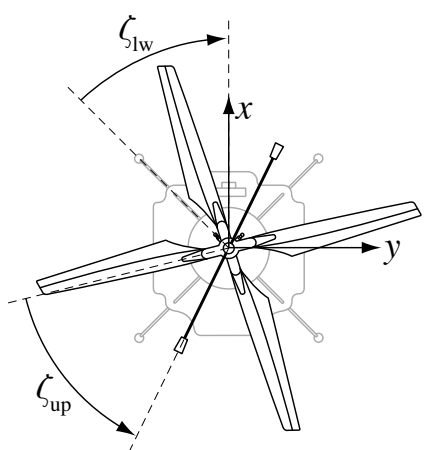

Fig. 2. The phase lag is the angle between application of a steering force and the point of maximal deflection. For the lower rotor it is the angle between the positioning of the servo motors and the main axis of the helicopter denoted as $\zeta_{\mathrm{lw}}$. For the upper rotor it is the angle between rotor and stabilizer bar $\zeta_{\text {up }}$.

We can express the angles of the upper rotor $\alpha_{\text {up }}$ and $\beta_{\text {up }}$ as a function of the stabilizer bar angles $\alpha_{\text {bar }}$ and $\beta_{\text {bar }}$ as

$$
\begin{aligned}
& \alpha_{\text {up }}=l_{\text {up }}\left(\alpha_{\text {bar }} \cos \left(\Delta \zeta_{\text {up }}\right)+\beta_{\text {bar }} \sin \left(\Delta \zeta_{\text {up }}\right)\right), \\
& \beta_{\text {up }}=l_{\text {up }}\left(\beta_{\text {bar }} \cos \left(\Delta \zeta_{\text {up }}\right)+\alpha_{\text {bar }} \sin \left(\Delta \zeta_{\text {up }}\right)\right) .
\end{aligned}
$$

The linkage factor $l_{\text {up }}$ accounts for the mechanical connection between the stabilizer bar and the upper rotor.

The point of the largest deflection of the stabilizer bar and the upper rotor are shifted in the direction of motion of the rotation due to the gyroscopic effect. This phase lag $\zeta_{\text {up }}$ is taken into consideration in the design of the rotor head by the connection between the stabilizer bar and the upper rotor with an angle $\zeta_{\text {up }}=49^{\circ}$ (fig. 2).

The phase lag is known to depend on the rotational speed [5], [6]. If the CoaX is used with a heavier payload, the rotational speed on the CoaX is higher and therefore the phase lag is not fully corrected by the mechanical offset. This offset of the phase lag $\Delta \zeta_{\text {up }}$ is taken into account in the model and can be approximated as

$$
\Delta \zeta_{\text {up }}=k_{\zeta, \text { up }} \Omega_{\text {up }}+o_{\zeta, \text { up }}
$$

with $k_{\zeta \text {,up }}$ and $o_{\zeta \text {,up }}$ being constants to be identified.

2) Lower Rotor and Swashplate: The swashplate is modelled the same way as the stabilizer bar. The cyclic input is now controlled by the servo motors which are mechanically shifted by $\zeta_{\text {lw }}=45^{\circ}$ (fig. 2).

$$
\begin{aligned}
\dot{\alpha}_{\mathrm{lw}}= & \frac{1}{\tau_{f, \mathrm{lw}}}\left(-\alpha_{\mathrm{lw}}\right. \\
& +l_{\mathrm{lw}}\left(u_{\mathrm{serv} 1} \cos \left(\Delta \zeta_{\mathrm{lw}}\right)-u_{\mathrm{serv} 2} \sin \left(\Delta \zeta_{\mathrm{lw}}\right)\right) \\
& \left.-a_{\alpha, v} v^{2} \operatorname{sign}(v)-a_{\alpha, u} u^{2} \operatorname{sign}(u)-a_{\alpha, p} p^{2} \operatorname{sign}(p)\right) \\
\dot{\beta}_{\mathrm{lw}}= & \frac{1}{\tau_{f, \mathrm{lw}}}\left(-\beta_{\mathrm{lw}}\right. \\
& +l_{\mathrm{lw}}\left(-u_{\mathrm{serv} 2} \cos \left(\Delta \zeta_{\mathrm{lw}}\right)+u_{\mathrm{serv} 1} \sin \left(\Delta \zeta_{\mathrm{lw}}\right)\right) \\
& \left.+a_{\beta, u} u^{2} \operatorname{sign}(u)-a_{\beta, v} v^{2} \operatorname{sign}(v)-a_{\beta, q} q^{2} \operatorname{sign}(q)\right)
\end{aligned}
$$

The time constant $\tau_{f, \text { lw }}$ covers all the dynamics by the servo motors and the lower rotor. The terms depending on the velocities $u$ and $v$ account for the effect of different air inflow on the rotors due to the speed stability and the conning effect, which are described in [2], [6], [7] and [8] respectively. The terms depending on the angular rates $p$ and $q$ describe the rotor damping [2], [7].
3) Drive Train: The upper drive train consists of the rotational dynamics of the stabilizer bar, the upper rotor, the upper drive shaft, the gear and the motor. As lower drive train the lower rotor, the swashplate, the lower drive shaft, the gear and the motor are considered. The rotational speeds additionally depend on the voltage of the battery $U_{\text {bat }}$.

We can describe this relationship as

$$
\begin{aligned}
\dot{\Omega}_{i}= & -\frac{1}{T_{d, i}} \Omega_{i}+\frac{1}{T_{d, i}}\left(k_{d, i} u_{\mathrm{mot}, i}+o_{d, i}\right) \\
& +\frac{1}{T_{d, i}} k_{d, U}\left(U_{\text {bat }}-U_{\text {norm }}\right),
\end{aligned}
$$

with $k_{d, i}, o_{d, i}$ and $k_{d, U}$ being constants.

\section{Model Summary}

The full nonlinear model consists of 18 states $\underline{x}$ and 4 inputs $\underline{u}$ ( 2 motors and 2 servo motors):

$$
\begin{aligned}
& \underline{x}=\left[\begin{array}{c}
x, y, z, u, v, w, \phi, \theta, \psi, p, q, r, \cdots \\
\cdots \alpha_{\mathrm{lw}}, \beta_{\mathrm{lw}}, \eta_{\mathrm{bar}}, \zeta_{\mathrm{bar}}, \Omega_{\mathrm{lw}}, \Omega_{\mathrm{up}}
\end{array}\right]^{\top}, \\
& \underline{u}=\left[u_{\mathrm{mot}, \mathrm{lw}}, u_{\mathrm{mot}, \mathrm{up}}, u_{\mathrm{serv} 1}, u_{\mathrm{serv} 2}\right]^{\top} .
\end{aligned}
$$

An overview of the model and the interconnections is given in fig. 3. Detailed equations are given in [4] and a MAT$\mathrm{LAB} /$ Simulink model is available from Skybotix.

\section{PARAMETER IDENTIFICATION}

The parameter identification consists of three different methods of how the unknown parameters are obtained. First, the mechanical parameters like the mass, inertia, center of gravity or the distance between the rotor hub and the CoG are directly measured on the real system or taken from the CAD model. For most of the aerodynamic parameters, a custom built test bench is used (sect. IV-A). Remaining parameters like the rotor damping have to evaluated during real flight and obtained with flight data recorded with a vision-based tracking system (sect. IV-B). Refer to [4] for a table with all parameters and their corresponding values.

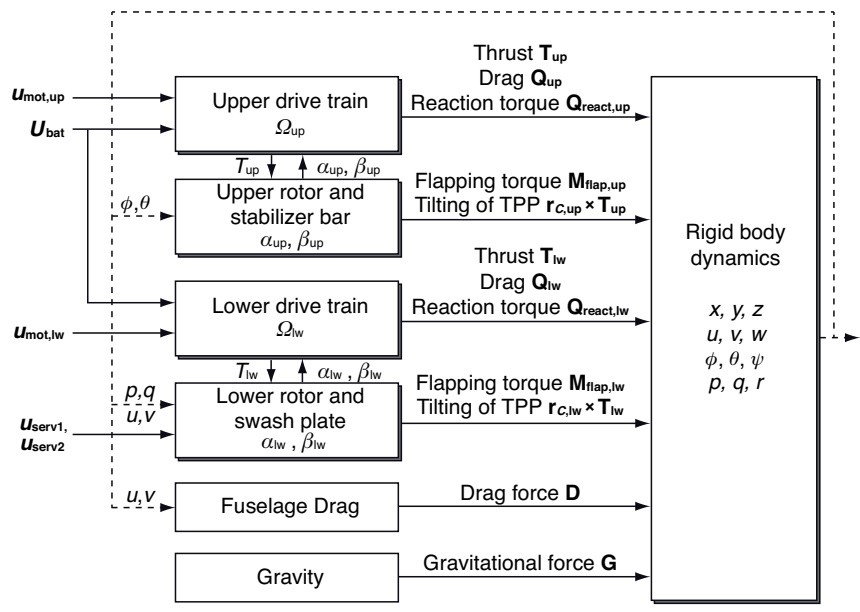

Fig. 3. The CoaX dynamic model block diagram shows the relevant forces and torques acting on the fuselage and their interconnections. The variables inside the blocks denote the state variables of the dynamic subsystems. 

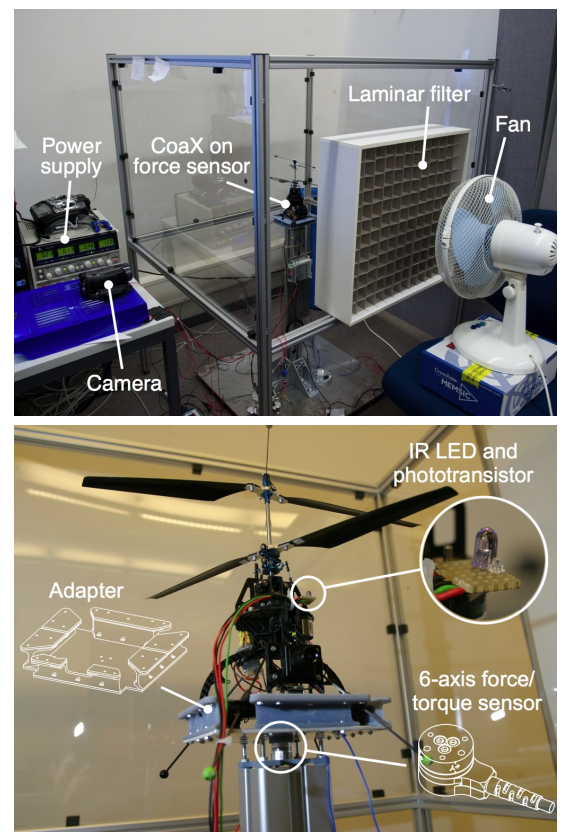

Fig. 4. Setup of the custom built test bench for the parameter identification of the CoaX. The test bench allows for independent analysis of the dynamics of the system. A fan is used to imitate the effect of high forward velocities.

Fig. 5. Close-up of the CoaX mounted on the test bench. Measurements are taken with a 6-axis force/torque sensor. The rotation speed of the rotors is measured with a combination of a IR LED, reflective markers and a phototransistor.

\section{A. Parameter Identification on the Test Bench}

The setup of the test bench is shown in fig. 4 and 5. The CoaX is mounted with an adapter on a commercial six-axis force/torque sensor. It is used to measure the forces $F_{x}$, $F_{y}, F_{z}$ and the torques $M_{x}, M_{y}, M_{z}$ on the CoG of the helicopter in the body frame. A rigid aluminum beam $\sim 1 \mathrm{~m}$ above ground carries the setup to eliminate the influence of the ground effect [9]. An infrared based tachometer is used for the measurement of the rotational speed of the rotors in combination with small pieces of reflective film attached to the rotors. In order to measure the effect of forward velocity a fan delivers airflow through a laminar filter to minimize turbulences. The wind speed is measured with a hot wire anemometer at the position where the airflow reaches the helicopter. For repeatable results the $\mathrm{CoaX}$ is attached to an external power supply with constant voltage.

1) Thrust and Drag: In a first step we analyze the relationship between the input to the motors $u_{\text {mot,lw }}$ and $u_{\text {mot,up }}$ and the rotational speeds $\Omega_{\mathrm{lw}}$ and $\Omega_{\text {up }}$ (in steady state and at a constant supply voltage). The input to one motor is varied over its whole range while the other motor is kept at hover condition. With these measurements the values for the gain $k_{d, i}$ and offset $o_{d, i}$ of the first order model of the drive train can be obtained precisely [4].

We have identified the thrust coefficients $c_{T, i}$ and drag coefficients $c_{Q, i}$ for the coaxial rotor setup with static mutual interference. ${ }^{1}$ In this configuration solely the total thrust and drag can be measured. Again, the rotational speed of one rotor is varied while the other is kept at hover condition.

2) Drive Train Dynamics: The dynamic of the drive trains are modeled as first order element (sect. III-C). For the identification of the time constants $\tau_{d, l w}$ and $\tau_{d, u p}$ the response of the rotors to step inputs on the motors is measured. A deeper analysis is given in [4].

\footnotetext{
${ }^{1}$ An analysis on the influence of the coaxial configuration in comparison to a single rotor setup is given in [4].
}

3) Phase Lag: As described in sect. III-C the mechanical design of the rotor head accounts for the phase lag. However, the phase lag changes as a function of the rotational speed. The phase lag angle can be measured as a function of the torques $M_{x}$ and $M_{y}$. The offset to pitch input on the swashplate is given by $\Delta \zeta=\operatorname{atan}\left(M_{x} / M_{y}\right)$. We have identified the factor $k_{\zeta, \mathrm{lw}}$ in the linear relation of the rotational speed and the phase lag and $k_{\zeta \text {,up }}=k_{\zeta \text {,lw }}$ is assumed. The offset parameters $o_{\zeta, \mathrm{lw}}$ and $o_{\zeta, \mathrm{up}}$ are identified later with the analysis of the flight data (sect. IV-B), due to imprecise trimming of the helicopter and structural bending of the helicopter at high forces on the test bench.

4) Swashplate: The torque on the fuselage resulting from steering input by the swashplate consists of the thrust induced torques $\mathbf{r}_{C} \times \mathbf{T}$ (tilting of the TPP) and the rotor flapping torque $\mathbf{M}_{\text {flap }}$ (sect. III-B). We can identify their contribution to the total torque by using the geometrical relationship

$$
M_{\text {flap }}=\sqrt{M_{x}^{2}+M_{y}^{2}}-z_{\mathrm{lw}} \sqrt{F_{x}^{2}+F_{y}^{2}}
$$

with $z_{\mathrm{lw}}$ denoting the distance between the $\mathrm{CoG}$ and the lower rotor hub (fig. 6). With this data we can easily identify the values of the lower blade stiffness $k_{\text {sprg,lw }}$ and the linkage factor between swashplate and lower rotor $l_{\text {lw }}$ (sect. III-C).

The dynamics of the swashplate and the lower rotor depend on the time constant $\tau_{f, \mathrm{lw}}$. We identify this parameter by applying step inputs on the servos and analyze the response based on the data from the force/torque sensor.

5) Upper Rotor and Stabilizer Bar: The stabilizer bar counteracts fast roll and pitch movements and its dynamics can therefore not be fully analyzed on the static test bench. Nevertheless, we identify the linkage factor between stabilizer bar and upper rotor $l_{\text {up }}$ by deflecting the stabilizer bar manually with a magnet (fig. 7).

The time constant $\tau_{f \text {,up }}$ of the first order approximation is identified later with the analysis of the flight data (sect. IV-B).

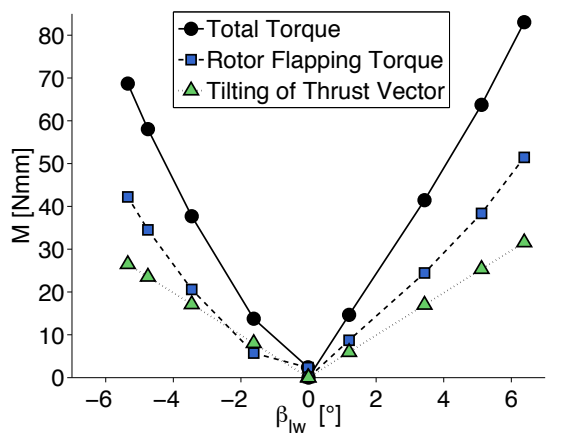

Fig. 6. Total torque caused by cyclic input as a function of the angle of the TPP $\beta_{\text {lw }}$. The total torque is the sum of the thrust induced torque (tilting of the TPP) and the flapping torque.

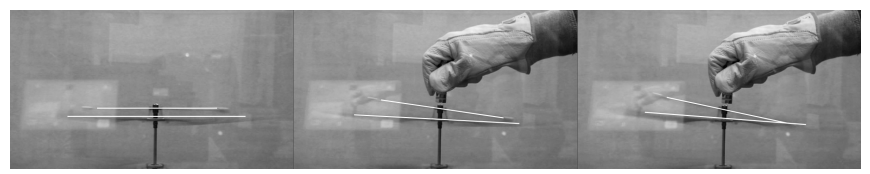

Fig. 7. In order to measure the linkage factor between stabilizer bar and upper rotor $l_{\text {up }}$ the stabilizer bar is deflected with a magnet 
6) Fuselage Drag: The velocity of the helicopter leads to a drag force because of the interaction of the fuselage with the air (indoor usage). We have measured this force by imitating the airflow with a fan and identified the parameters $a_{\mathrm{Fx}, u}$ and $a_{\mathrm{Fy}, v}$ of the drag force $\mathbf{D}$ in (7).

7) Battery Voltage: The rotational speed depends on the voltage of the power source. This relationship is included in the model (sec. III-C) and the parameter $k_{d, U}$ is found by varying the power supply voltage from 10.5 to $12.5 \mathrm{~V}$.

\section{B. Parameter Identification on Flight Data}

The recording of real flight data serves two reasons. First, the parameters that cannot be extracted from measurements on the test bench can be identified on flight data. Second, the model can be validated by comparing the simulation results to the real data when the identical input is used (sect. IV-C).

For recording the flight data, the CoaX was steered by a pilot. Additionally, the onboard altitude and yaw control is used to help the pilot. Effectively, the pilot has full control over the pitch and roll dynamics (servo motors) and determines the set point values for the yaw and height control (motors). The true input values to the servo motors and motors is recorded on the CoaX at $100 \mathrm{~Hz}$. A Vicon motion capture system is used to precisely record the full position and attitude at $200 \mathrm{~Hz}$. The ETH Flying Machine Arena [10] consists of a $10 \times 10 \times 10 \mathrm{~m}$ space equipped with 8 cameras. The tracking data and the data logged on the CoaX were synchronized by matching distinctive roll/pitch/ yaw movements at the beginning and at the end of the tests.

The parameters that are identified on flight data are namely $a_{\alpha, v}, a_{\alpha, u}, a_{\alpha, p}, \tau_{f \text {,up }}, o_{\zeta, \text { up }}$ and $o_{\zeta, \text { lw }}$. For the other parameters we can assume $a_{\beta, u}=a_{\alpha, v}, a_{\beta, v}=a_{\alpha, u}$ and $a_{\beta, q}=a_{\alpha, p}$ thanks to the symmetry of the CoaX. As data for the identification process a sweep input on the pitch axis is applied on the system. The input was conducted by the pilot manually and has a frequency range of $\sim 0.5-8 \mathrm{~Hz}$. The parameters are identified by comparing the real response to the results of the simulation and minimizing the least squares error with MATLAB's fminsearch function. Results and a list of all parameter values are given in [4].

\section{Model Validation}

We have recorded flight data of the CoaX with the motion capture system to compared it to the response of the model. ${ }^{2}$ The results for the horizontal motion is shown in fig. 8 . Generally, the model shows a satisfactory agreement with the system behavior. A good correlation on the main axis (with respect to the input $u_{\text {serv }}$ ) can be observed while the lateral axis is slightly off. Figure 9 shows the vertical dynamics of the CoaX. The correlation between model prediction, and data obtained during the flight test is satisfactory and the model captures the dynamics reasonabely.

\section{Decoupling Control}

The goal of the control here is to decouple the dynamics of the CoaX in order to enable easier and more precise

\footnotetext{
${ }^{2}$ This set of data was not used for any identification process.
}

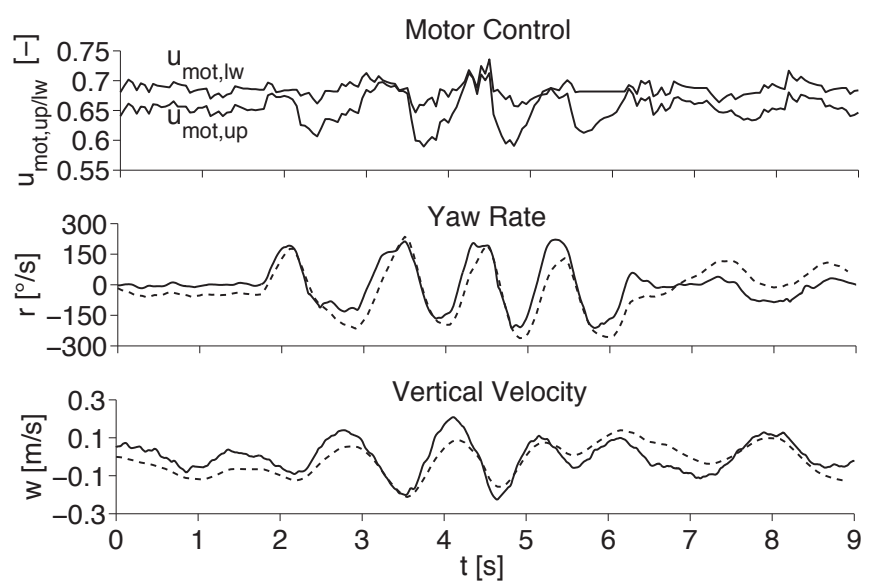

Fig. 9. Comparison between the responses obtained during flight tests (solid) and the responses predicted by the identified model (dashed). The flight data is recorded with a vision based motion tracking system.

operation. Based on our analysis, three main coupling effects are observable.

1) Coupling between the roll/pitch dynamics and the altitude dynamics: By changing the direction of the thrust vector with cyclic input on the rotors or by rolling/pitching the fuselage, the vertical component of the thrust vector decreases. This leads to an unbalance between the gravitational force and the thrust force, leading to undesired loss of the height of the helicopter.

2) Coupling due to a not corrected phase lag of the upper rotor and stabilizer bar: An angle between upper rotor and stabilizer bar which is not adjusted to the mean rotational speed causes the stabilizer bar to correct off the axis of the disturbance. This leads to a unwanted oscillation of the helicopter.

3) Coupling between longitudinal and lateral dynamics:

The helicopter follows distorted trajectories on steering inputs if the positioning of the servo motors to the actual phase lag of the lower rotor is not adjusted. Also, the different airflow on the rotor blades at high velocities leads to stimulation of the off-axis dynamics. These effects manifest themselves in a unsatisfying directional constancy.

The effect of 1) is compensated sufficiently by a simple height-controller (PI with feed forward). The effect of 2) is usually negligible for a small upper phase lag offset ( $\Delta \zeta_{\text {up }} \cong 7^{\circ}$ for our CoaX configuration). We focus on 3 ) because it causes the biggest disturbances on horizontal trajectories. The problem is solvable with a non-linear input transformation based on the current state. We use (13) and (14) to derive the equations for the decoupling controller:

$$
\begin{aligned}
\hat{u}_{\text {serv } 1}= & \frac{1}{\cos \left(\Delta \zeta_{\text {lw }}\right)}\left(u_{\text {serv } 1}\right. \\
& \left.+u_{\text {serv } 2} \sin \left(\Delta \zeta_{\text {lw }}\right)+u^{2} \frac{a_{\alpha, u}}{l_{\mathrm{lw}}} \operatorname{sign}(u)\right), \\
\hat{u}_{\text {serv } 2}= & \frac{1}{\cos \left(\Delta \zeta_{\text {lw }}\right)}\left(u_{\text {serv } 2}\right. \\
& \left.+u_{\text {serv } 1} \sin \left(\Delta \zeta_{\text {lw }}\right)-v^{2} \frac{a_{\beta, v}}{l_{\text {lw }}} \operatorname{sign}(v)\right) .
\end{aligned}
$$



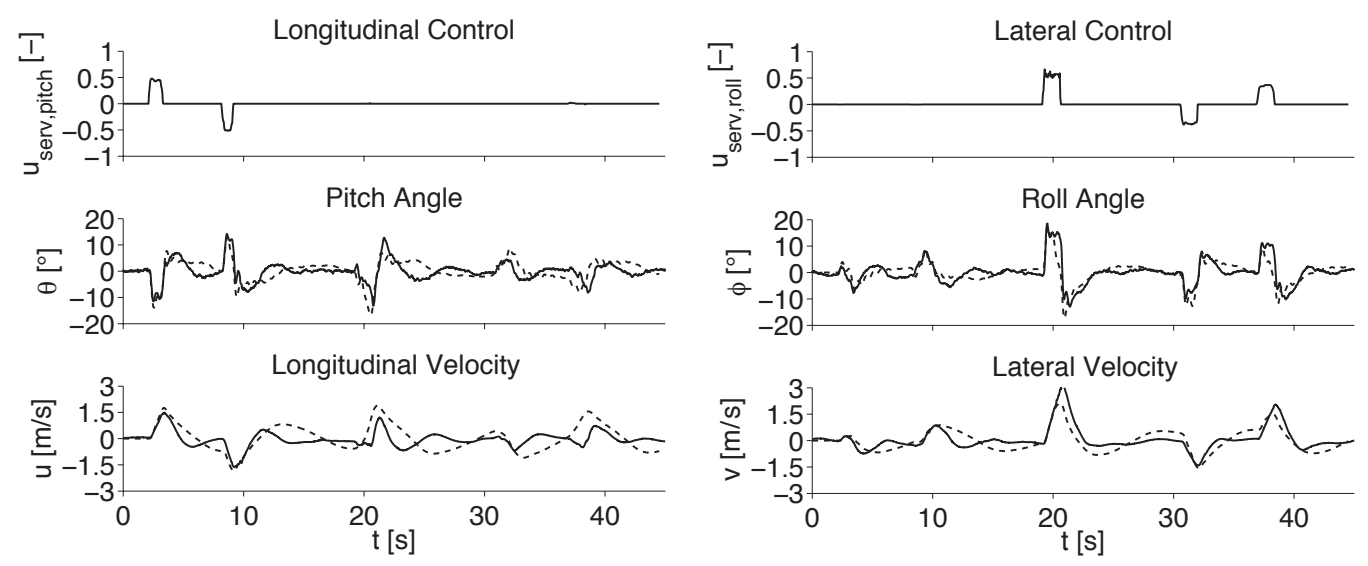

Fig. 8. Comparison between the responses obtained during flight tests (solid) and the responses predicted by the identified model (dashed). The flight data is recorded with a vision based motion tracking system. The influence of the coupling effects is visible in the dynamics of lateral axis with respect to the input $u_{\text {serv }}$.

The variables $\hat{u}_{\text {serv1 }}$ and $\hat{u}_{\text {serv2 }}$ denote the decoupled inputs to the servo motors. The phase lag offset of the lower rotor $\Delta \zeta_{\text {lw }}$ can be approximated by a constant at hover condition or calculated with

$$
\begin{aligned}
\Delta \zeta_{\mathrm{lw}} & =k_{\zeta, \mathrm{lw}} \cdot \Omega_{\mathrm{lw}}+o_{\zeta, \mathrm{lw}}, \\
\Omega_{\mathrm{lw}} & =k_{d, \mathrm{lw}} \cdot u_{\mathrm{mot}, \mathrm{lw}}+o_{d, \mathrm{lw}}+k_{d, U} \cdot\left(U_{\mathrm{bat}}-U_{\mathrm{norm}}\right) .
\end{aligned}
$$

We test this controller on the CoaX in a mode in which only the height and yaw control is active. The decoupling controller is applied to the manual input for roll/pitch. The CoaX is trimmed manually in hover. The velocity is estimated by the the CoaX speed module which uses a mouse sensor to estimate the horizontal velocity [11]. Figure 10 and the video attachment show the comparison between the dynamics with and without decoupling controller. A longitudinal step input is applied. The controller actively compensates for the coupling in the longitudinal and lateral dynamics and produces a satisfactory precision in the motion of the CoaX.

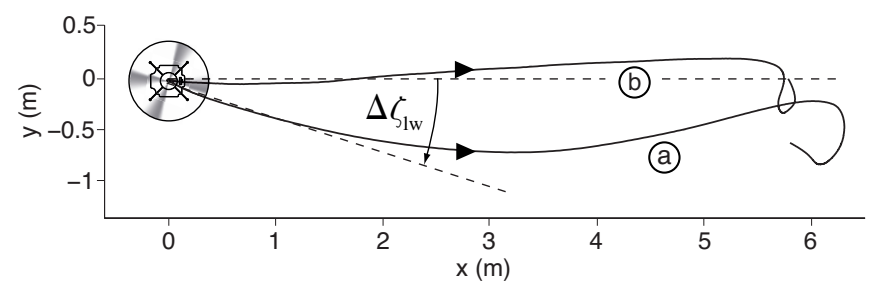

Fig. 10. Comparison between the trajectories of the CoaX on a longitudinal step input (max. velocity $1.8 \mathrm{~m} / \mathrm{s}$ ). The trajectory (a) shows the motion without decoupling controller and the trajectory (b) shows the motion with active decoupling controller. The initial direction offset is due to the phase lag offset $\Delta \zeta_{\text {lw }}$. The coupling effect at higher speed is due to irregular airflow on the blades. An associated video is available as attachment to this paper.

\section{SUMmARY AND FUtURE WORK}

This paper shows the development of a nonlinear model for a coaxial micro helicopter based on gray-box modeling and parameter identification. The model accounts for hover and cruise flight situations and explicitly captures the offaxis dynamics. This makes it suitable for the simulation and analysis of dynamic flight maneuvers. The experiments on the test bench allow for valuable insight into the dynamics of the system and they are essential for the main part of the parameter identification. The recording of flight data with a vision based tracking system allows for additional parameter identification. Furthermore, the model is validated successfully on the basis of a different set of flight data. A decoupling controller is developed and implemented which shows to increase the accuracy in the flight behavior of the CoaX.

The model will be used in the future for real-time simulations of the CoaX. The decoupling controller will help in different applications and allow for easier handling and more precise control of the CoaX. A full, model-based control for all DOF of the helicopter will be implemented. However, this would require enough computational time for a state observer of the states that cannot directly be measured. An interesting approach for increasing the dynamic performance for aggressive flight maneuvers is to remove the stabilizer bar, although this would require fast sensors and actuators.

\section{REFERENCES}

[1] D. Schafroth, C. Bermes, S. Bouabdallah, R. Siegwart, "Modeling and System Identification of the muFly Micro Helicopter", Journal of Intelligent and Robotic Systems, 2009.

[2] B. Mettler, Identification Modeling and Characteristics of Miniature Rotorcraft, Kluwer Academic, Dordrecht, 2003.

[3] R. Siegwart, S. Bouabdallah, S. Leutenegger, S. Weiss, Unmanned Aircraft Design, Modeling and Control, Lecture Notes, Lecture 2, 2010, ETH Zurich.

[4] P. Fankhauser, Modeling and Control of the CoaX Micro Helicopter, Semester Thesis, ETH Zurich, 2011.

[5] A. Bramwell, Helicopter Dynamics, 2nd edn. Butterworth Heinemann, Boston, 2001.

[6] J. Watkinson, The Art of the Helicopter, Elsevier, Amsterdam, 2004.

[7] G. D. Padfield, Helicopter Flight Dynamics: The Theory and Application of Flying Qualities and Simulation Modeling, AIAA Education Series, 1996.

[8] R. W. Prouty, Helicopter Aerodynamics, PJS Publication Inc., 1985.

[9] V. Gresch, Development of a Test Bench for the AIRobots Inspection Rotorcraft, Semester Thesis, ETH Zurich, 2010.

[10] S. Lupashin, A. Schöllig, M. Sherback, R. D'Andrea, "A simple learning strategy for high-speed quadrocopter multi-flips", Robotics and Automation (ICRA), 2010 IEEE International Conference, 16421648, 2010.

[11] P. Gohl and M. Egli, Vision-Based Hovering with a Micro Helicopter, Semester Thesis, ETH Zurich, 2009. 